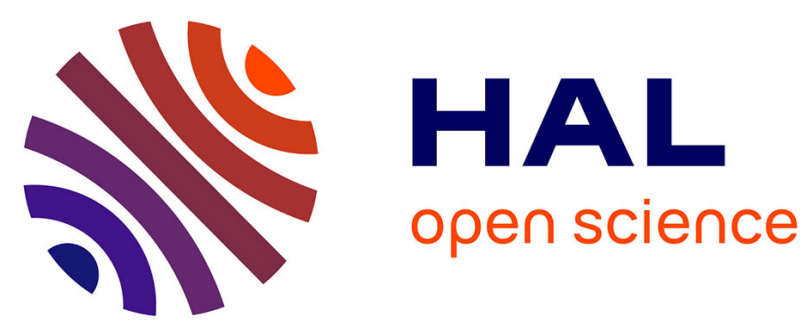

\title{
Singular constant control trajectories and transition degeneracies in a closed 4-level quantum system
}

Andreea Grigoriu, Mohamed Belhadj, Gabriel Turinici

\section{To cite this version:}

Andreea Grigoriu, Mohamed Belhadj, Gabriel Turinici. Singular constant control trajectories and transition degeneracies in a closed 4-level quantum system. 49TH IEEE CONFERENCE ON DECISION AND CONTROL (CDC), Atlanta 2010, Dec 2010, Atlanta (GA), United States. pp.5444-5449, 10.1109/CDC.2010.5717900 . hal-00522130

\section{HAL Id: hal-00522130 \\ https://hal.science/hal-00522130}

Submitted on 29 Sep 2010

HAL is a multi-disciplinary open access archive for the deposit and dissemination of scientific research documents, whether they are published or not. The documents may come from teaching and research institutions in France or abroad, or from public or private research centers.
L'archive ouverte pluridisciplinaire HAL, est destinée au dépôt et à la diffusion de documents scientifiques de niveau recherche, publiés ou non, émanant des établissements d'enseignement et de recherche français ou étrangers, des laboratoires publics ou privés. 


\title{
Singular constant control trajectories and transition degeneracies in a closed 4-level quantum system
}

\author{
Mohamed Belhadj, Andreea Grigoriu and Gabriel Turinici
}

\begin{abstract}
We analyze in this paper controllability aspects for a 4-dimensional quantum system. The "strong regularity" (cf. [1]) has been proven to be sufficient condition for global controllability. A conjecture in the literature asks whether this condition (up to the introduction of an additive constant in the control field) is also necessary. We prove here a negative result.

The result also applies to the study of singularity of trajectories associated to constant control fields. The theoretical result is supported by numerical simulations.
\end{abstract}

\section{INTRODUCTION}

The development of the laser technology in the 60's brought into the attention of the scientific community the possibility to use the lasers to control chemical reactions at a quantum level [2], [4], [6], [8], [9], [12]. Latter the control of quantum system has been extended to other types of interaction such as magnetic fields in NMR experiments. The interest related to this technique is that the contactless manipulation affects the very structure of molecules and has the potential to allow precision far beyond the usual macroscopic means (temperature, pressure,...).

Today the technique has many other applications [6] in various fields: designing logical gates for the next generation of quantum computers, medical imaging by nuclear magnetic resonance (NMR), study of protein dynamics, molecular detection, molecular orientation and alignment, construction of ultra-short laser.

The first experiments proved that controlling quantum phenomena by external fields is a rather difficult task to handle that the physical intuition alone cannot accomplish. This hinted to the necessity of introducing rigorous control theory tools. An important preliminary of experimental implementation is the study of the feasibility through theoretical methods and computer simulations. In such a context informations on the controllability of the system are crucial to the future success of the experiment.

Depending on the application, the field that manipulates the quantum system can have tendency to rather be oscillatory (as in femtosecond chemistry when the laser is highly

This work was supported by INRIA Rocquencourt projet MicMac and OMQP, from "Agence Nationale de la Recherche" (ANR), Projet Blanc C-QUID number BLAN-3-139579, from PICS NSF-CNRS and from IFC (Institut Français de Coopération).

M. Belhadj Institut Supérieur des Mathématiques Appliquées et d'Informatique de Kairouan, Av. Assad Ibn El Fourat, 3100 Kairouan, Tunisie. mohamed.belhadjeismai.rnu.tn

A. Grigoriu CEREMADE, Université Paris-Dauphine, Place du Maréchal de Lattre de Tassigny, 75016, Paris, France. grigoriulceremade.dauphine.fr

G. Turinici CEREMADE, Université Paris-Dauphine, Place du Maréchal de Lattre de Tassigny, 75016, Paris, France. gabriel.turiniciedauphine.fr oscillatory) or on the contrary constant (magnetic fields in NMR applications).

The controllability has been investigated in the context of the bilinear systems on Lie groups and several criterions have been put forward [1], [11].

Controllability can be of two types: local and global. Passing from local to global controllability is a delicate task and compactness is one of the arguments that can be invoked. Several questions are of interest: around which trajectories does local controllability hold, when is the system globally controllable, etc.

In finite dimensional systems, global controllability has been proved when the system is "strongly regular" cf. [1]. However the "strong regularity" is only a sufficient condition and a natural question arises: can any controllable system be proven to be strongly regular (eventually after a shift in the field). This has been formulated as a conjecture in the literature. The main result gives a negative answer to the posed question if the dimension of the system is larger than 4.

On the other hand we analyse the local controllability around a constant field. When local controllability does not hold in the neighborhood of a field we say that the corresponding trajectory is singular. We show in this paper that systems exist such that all constant fields give rise to singular trajectories.

The balance of the paper is as follows: in section II the general control concepts and a detailed motivation of the article are introduced, in section III the theoretical result is presented supported by some numerical tests in section IV.

\section{General Setting}

We consider a quantum system evolving under the Schrödinger equation (we use atomic units, i.e $\hbar=1$ ):

$$
\begin{aligned}
i \frac{\partial}{\partial t} \Psi(x, t) & =H(t) \Psi(x, t) \\
\Psi(x, t=0) & =\Psi_{0}(x),
\end{aligned}
$$

where $\Psi(x, t)$ is the wave-function, $\Psi_{0}(x)$ is the initial data, $x \in \mathbb{R}^{\gamma}$ the internal set of degrees of freedom and $H(t)$ the Hamiltonian of the system, a self adjoint operator over $L^{2}\left(\mathbb{R}^{\gamma}\right)$ (i.e $H(t)=H^{\dagger}(t)$ ).

In the presence of an external field the evolution of the system can be modeled by:

$$
\begin{aligned}
i \frac{\partial}{\partial t} \Psi(x, t) & =\left(H_{0}+\epsilon(t) H_{1}\right) \Psi(x, t) \\
\Psi(x, t=0) & =\Psi_{0}(x),
\end{aligned}
$$


where $H_{1}$ is the operator connecting the system with the laser field $\epsilon(t) \in \mathbb{R}$ and $H_{0}$ is the internal Hamiltonian.

The $L^{2}$ norm is conserved during the evolution:

$$
\|\Psi(x, t)\|_{L^{2}\left(\mathbb{R}^{\gamma}\right)}=\left\|\Psi_{0}\right\|_{L^{2}\left(\mathbb{R}^{\gamma}\right)} \quad \forall t>0
$$

and the state belongs to the unit sphere $\mathcal{S}(0,1)$ of $L^{2}\left(\mathbb{R}^{\gamma}\right)$ :

$$
\mathcal{S}(0,1)=\left\{f \in L^{2}\left(\mathbb{R}^{\gamma}\right) ;\|f\|_{L^{2}\left(\mathbb{R}^{\gamma}\right)}=1\right\} .
$$

In order to avoid trivial cases we suppose $\left[H_{0}, H_{1}\right] \neq 0$, where the Lie bracket $[\cdot, \cdot]$ is defined as: $[X, Y]=X Y-Y X$.

We work in a finite dimensional setting and consider a Galerkin discretization of the Time Dependent Schrödinger Equation (2). We take the basis functions $\left\{\varphi_{i} ; i=1, \ldots, N\right\}$, e.g. the eigenfunctions of $H_{0}: H_{0} \varphi_{k}=e_{k} \varphi_{k}$, the wavefunction is written as: $\Psi=\sum_{j=1}^{N} c_{j} \varphi_{j}$. We denote by $A$ and $B$ the matrices $(N \times N)$ associated to the operators $H_{0}$ and $H_{1},(A)_{k l}=\left\langle\varphi_{k} \mid H_{0} \varphi_{l}\right\rangle,(B)_{k l}=\left\langle\varphi_{k} \mid H_{1} \varphi_{l}\right\rangle$, for $k, l$ from 1 to $N$. For simplicity we preserve the notation $\Psi$ for the wave-function, $\Psi=\left(c_{j}\right)_{j=1}^{N}$.

Here the symbol $|\varphi\rangle$ represents the bra-ket notation, $\langle\varphi|$ it's adjoint state and $\langle\cdot \mid \cdot\rangle$ the hermitian product.

We obtain the $N$ - dimensional system:

$$
\begin{aligned}
i \frac{d}{d t} \Psi(t) & =(A+\epsilon(t) B) \Psi(t) \\
\Psi(t=0) & =\Psi_{0},
\end{aligned}
$$

where $\Psi_{0}=\left(c_{j}^{0}\right)_{j=1}^{N}$. The property of norm conservation is preserved:

$$
\sum_{j=1}^{N}\left|c_{j}\right|^{2}=1 .
$$

In the following without loss of generality we suppose that $A$ is a diagonal matrix and $B$ a real symmetrical matrix (Hermitian). We conserve the assumption $[A, B] \neq 0$, initially introduced for the operators $H_{0}$ and $H_{1}$, in order to avoid trivial control problems.

\section{A. Global controllability and strongly regular systems}

In order to prove the controllability of the finite dimensional system (5), we can use the Lie algebra rank condition: the system (5) is controllable if the Lie algebra generated by $i A$ and $i B$, denoted by $\mathcal{L}_{A, B}$ has the dimension $N^{2}$ (or $N^{2}-1$ if $i A$ and $i B$ are traceless).

Recall that the Lie algebra generated by $i A$ and $i B$ is the real vector space spanned by all the combinations of commutations of $i A$ and $i B$ and their iterations, for example $[i A,[i A, i B]]$, where $[i A, i B]=(i A)(i B)-(i B)(i A)=$ $B A-A B$.

Although it is a very elegant way to prove controllability, for large values of $N$ this criteria becomes difficult to check (and there is no intuition to explain the result once obtained). In the literature we can find other theoretical results [10], [11], based on graph theory, that give necessary conditions to prove that the system (5) is controllable. Since one of this results [1], [10], [11] is relevant for our paper we present it in the following.
We define the graph $G=(V, E)$, where $V$ is the set of vertices and $E$ the set of edges, as follows:

$$
\begin{aligned}
G=(V, E): \quad V & =\left\{\varphi_{i}, i=1 \ldots N\right\}, \\
& E=\left\{\left(\varphi_{i}, \varphi_{j}\right), i<j, B_{i j} \neq 0\right\}
\end{aligned}
$$

We denote by: $\omega_{i j}=\mu_{i}^{0}-\mu_{j}^{0}, i, j=1, \ldots, N$, the eigenvalues difference for the matrix $A$.

Theorem 2.1: Under the hypothesis:

$H_{1}$ : The graph $G$ is connected

$H_{2}$ : The graph $G$ doesn't have degenerate transitions, i.e for all $(i, j) \neq(a, b) i \neq j, a \neq b$ such that $B_{i j} \neq 0$, $B_{a b} \neq 0, \omega_{i j} \neq \omega_{a b}$

the system (5) is controllable, that is for any $\Psi \in \mathcal{S}^{N}(0,1)$ the set of reachable sets is $\mathcal{S}^{N}(0,1)$, where $\mathcal{S}^{N}(0,1)$ is the unit sphere of $\mathbb{C}^{N}$.

A system that satisfy hypothesis above is called "strongly regular" [1] or again "with non-degenerate transitions" [11]. This class of systems has very nice mathematical properties: the Lie algebra spanned by $i A, i B$ can be constructed explicitly and the global controllability holds [1]; moreover the system is locally controllable around the null field for generically any initial state [11]. A subclass with stronger requirements (the "ideal systems") were showed to be important in the study of the Lyapunov stability [13].

Remark 2.1: The "strong regularity" is not a necessary condition for controllability in the wave-function formulation (but it is sufficient together with hypothesis $H_{1}$ ).

On the contrary if we consider the density matrix formulation of (5)

$$
\begin{aligned}
i \frac{d}{d t} \rho(t) & =(A+\epsilon(t) B) \rho(t) \\
\rho(t=0) & =\rho_{0},
\end{aligned}
$$

then hypothesis $\mathrm{H}_{2}$ is necessary.

There are systems, for example [5], [7] :

$$
\begin{aligned}
A & =\left(\begin{array}{cccc}
0 & 0 & 0 & 0 \\
0 & 0.04556 & 0 & 0 \\
0 & 0 & 0.095683 & 0 \\
0 & 0 & 0 & 0.095683
\end{array}\right) \\
B & =\left(\begin{array}{cccc}
0 & 1 & 1 & -1 \\
1 & 0 & 1 & 1 \\
1 & 1 & 0 & 0 \\
-1 & 1 & 0 & 0
\end{array}\right),
\end{aligned}
$$

for which the second hypothesis, is not fulfilled, but the system is still controllable. The explanation is the following ( see [10], [11] for more details) : take $\epsilon(t)=\lambda+\widetilde{\epsilon}(t)$, then the triplet $(A, B, \epsilon(t))$ is transformed into $(A+\lambda B, B, \widetilde{\epsilon}(t))$. We can find a unitary transformation $U(\lambda)$ such that $\widetilde{A}=$ $U(\lambda)(A+\lambda B) U^{t}(\lambda)$ is a diagonal matrix and the dipole matrix $\widetilde{B}=U(\lambda) B U^{t}(\lambda)$. Then the $\mathrm{N}$-dimensional systems (5), that models the evolution is equivalent to:

$$
\begin{aligned}
i \frac{d}{d t} \widetilde{\Psi}(t) & =(\widetilde{A}+\widetilde{\epsilon}(t) \widetilde{B}) \Psi(t) \\
\widetilde{\Psi}(t=0) & =U(\lambda) \Psi_{0} .
\end{aligned}
$$

In conclusion, a sufficient condition for controllability of the finite dimensional system (5) is the existence of $\lambda$ such that 
the system defined by the triplet $(A+\lambda B, B, \widetilde{\epsilon}(t))$ is strongly regular.

The natural question to be asked here is: suppose a system is controllable $(A, B)$, does it exist $\lambda$ such that $(A+\lambda B, B)$ is strongly regular? The situation for $N=3$ was analyzed in [14] (section A.3 page 163) and the answer is affirmative.

\section{B. Singular trajectories around constant fields}

When the fields are naturally constant (as in NMR) or piecewise constant the study of the singular trajectories around constant fields $\lambda$ can be formulated as: is the system

$$
\begin{aligned}
i \frac{d}{d t} \Psi(t) & =(A+\lambda B+\epsilon(t) B) \Psi(t) \\
\Psi(t=0) & =\Psi_{0},
\end{aligned}
$$

locally controllable?

We recall the definition of local controllable around a field $\epsilon(t)$ in a set $\mathcal{U}$, for a giving final state $\bar{\Psi}=\Psi(T)$ with $\epsilon(t)=\bar{\epsilon}(t)$ in equation (5).

Definition 2.1: The system (5) is called locally controllable if there exists neighborhoods $X$ of $\bar{\Psi}$ and $Y$ of $\bar{\epsilon}$ in $\mathcal{U}$ such that for any $\overline{\bar{\Psi}} \in X$ there exists $\overline{\bar{\epsilon}} \in Y$ such that the system (5) has $\Psi(T)=\overline{\bar{\Psi}}$.

The local controllability can be analyzed as in [11] Thm.1 by considering the matrix $A+\lambda B$ instead of the matrix $A$. If for all $\lambda \in \mathbb{R}$ the transitions of $A+\lambda B$ are degenerate (or equivalently $A+\lambda B$ is not strongly regular) then there exists no constant field around which the system will be locally controllable.

\section{THEORETICAL RESULT}

In the following by $s u(N)$ we understand the Lie algebra of $\mathrm{N}$-dimensional null trace hermitian matrices. We denote by $\left(\mu_{i}^{\lambda}\right)_{i=1, \ldots, N}$ the eigenvalues of $A+\lambda B$ and by $\left(\mu_{i}^{0}\right)_{i=1, . ., N}$ the eigenvalues of $A$, for example the energies corresponding to the states $\varphi_{i}$.

Theorem 3.1: There exists $A=\left(A_{i j}\right)_{i, j=1, \ldots, 4}$ a real diagonal matrix and $B=\left(B_{a b}\right)_{a, b=1, \ldots, 4}$ a symmetrical matrix, both 4-dimensional with the following properties:

(i) $[A, B] \neq 0$ (i.e $A B \neq B A$ )

(ii) The real Lie algebra generated by $i A$ and $i B$ contains $s u(4)$ (or $u(4)$ if $\operatorname{Tr}(A) \neq 0$ )

such that for every $\lambda \in \mathbb{R}$ there exists $(i, j) \neq(a, b), i \neq j$, $a \neq b$ with $B_{i j} \neq 0, B_{a b} \neq 0$ such that:

\section{Proof:}

$$
\mu_{i}^{\lambda}-\mu_{j}^{\lambda}=\mu_{a}^{\lambda}-\mu_{b}^{\lambda}
$$

Even if a a construction of a single type of matrix would be enough, the goal is to detect all the matrices that are candidate to satisfy the theorem.
Let us take $A$ a real diagonal matrix and $B$ a symmetrical matrix:

$$
\begin{aligned}
A & =\left(\begin{array}{cccc}
\mu_{1}^{0} & 0 & 0 & 0 \\
0 & \mu_{2}^{0} & 0 & 0 \\
0 & 0 & \mu_{3}^{0} & 0 \\
0 & 0 & 0 & \mu_{4}^{0}
\end{array}\right) \\
B & =\left(\begin{array}{cccc}
B_{11} & B_{12} & B_{13} & B_{14} \\
B_{12} & B_{22} & B_{23} & B_{24} \\
B_{13} & B_{23} & B_{33} & B_{34} \\
B_{14} & B_{24} & B_{34} & B_{44}
\end{array}\right) .
\end{aligned}
$$

Without loss of generality (see Remark A.2.3 page 169 in [14]) we can set $\operatorname{Tr}(A)=\operatorname{Tr}(B)=0$, we obtain

$$
\operatorname{Tr}(A+\lambda B)=\operatorname{Tr}(A)+\lambda \operatorname{Tr}(B)=0 \quad \forall \lambda \in \mathbb{R} .
$$

This is equivalent to:

$$
\mu_{1}^{\lambda}+\mu_{2}^{\lambda}+\mu_{3}^{\lambda}+\mu_{4}^{\lambda}=0
$$

From relation (14) for $\lambda=0$ and $\lambda=1$ we have:

$$
\begin{aligned}
\mu_{1}^{0}+\mu_{2}^{0}+\mu_{3}^{0}+\mu_{4}^{0} & =0 \\
B_{11}+B_{22}+B_{33}+B_{44} & =0
\end{aligned}
$$

- First case: A is non-degenerate

We say that $A$ is non-degenerate if for every $i \neq j$, $i, j=1, \ldots, 4, \mu_{i}^{0} \neq \mu_{j}^{0}$.

In this case lets us take

$$
\begin{aligned}
& \mu_{1}^{0}=-\eta, \mu_{2}^{0}=-\epsilon, \mu_{3}^{0}=\epsilon, \mu_{4}^{0}=\eta \\
& B_{11}=-B_{44}, B_{22}=-B_{33} .
\end{aligned}
$$

Thus $A$ and $B$ are defined as:

$$
\begin{aligned}
A & =\left(\begin{array}{cccc}
-\eta & 0 & 0 & 0 \\
0 & -\epsilon & 0 & 0 \\
0 & 0 & \epsilon & 0 \\
0 & 0 & 0 & \eta
\end{array}\right) \\
B & =\left(\begin{array}{cccc}
B_{11} & B_{12} & B_{13} & B_{14} \\
B_{12} & B_{22} & B_{23} & B_{24} \\
B_{13} & B_{23} & -B_{22} & B_{34} \\
B_{14} & B_{24} & B_{34} & -B_{11}
\end{array}\right),
\end{aligned}
$$

with $B_{13} \neq 0$ and $B_{24} \neq 0$.

We can easily note that for $\lambda=0$ equality (12) is fulfilled since there exists $(1,3) \neq(2,4), 1 \neq 3,2 \neq 4$ with $B_{13} \neq 0, B_{24} \neq 0$ such that

$$
\mu_{1}^{0}-\mu_{3}^{0}=\mu_{2}^{0}-\mu_{4}^{0}
$$

Since we are in the 4-dimensional case, relation (12) together with relation (15) is equivalent to the existence of $(i, j)$ and $(a, b)$ with $i \neq j, a \neq b, i, j, a, b=1, \ldots, 4$ such that:

$$
\begin{aligned}
& \mu_{i}^{\lambda}=-\mu_{b}^{\lambda} \\
& \mu_{j}^{\lambda}=-\mu_{a}^{\lambda}
\end{aligned}
$$

We consider the polynomial:

$$
\begin{aligned}
P(X)= & \left(X-\mu_{1}^{\lambda}\right)\left(X-\mu_{2}^{\lambda}\right) \\
& \left(X-\mu_{3}^{\lambda}\right)\left(X-\mu_{4}^{\lambda}\right) .
\end{aligned}
$$


If relation $(20)$ is fulfilled for $(i, j)=(1,3)$ and $(a, b)=(2,4)$ then:

$$
\begin{aligned}
P(X)= & X^{4}-X^{2}\left(\left(\mu_{1}^{\lambda}\right)^{2}+\left(\mu_{2}^{\lambda}\right)^{2}\right)+ \\
& \left(\mu_{1}^{\lambda}\right)^{2}\left(\mu_{2}^{\lambda}\right)^{2} .
\end{aligned}
$$

The polynomial $P(X)$ is also defined by

$$
\begin{aligned}
P(X) & =\operatorname{det}\left(X I_{4}-(A+\lambda B)\right) \\
& =X^{4}+\alpha X^{2}-\beta X+\gamma
\end{aligned}
$$

where $I_{4}$ is the 4-dimensional identity matrix.

By identification we obtain the following system:

$$
\begin{aligned}
\alpha & =-\left(\left(\mu_{1}^{\lambda}\right)^{2}+\left(\mu_{2}^{\lambda}\right)^{2}\right) \\
\beta & =0 \\
\gamma & =\left(\mu_{1}^{\lambda}\right)^{2}\left(\mu_{2}^{\lambda}\right)^{2} .
\end{aligned}
$$

We compute $\operatorname{det}\left(X I_{4}-(A+\lambda B)\right)$ by replacing $A$ and $B$ with (18) and we obtain $\beta$, a third order polynomial in $\lambda$. We want $\beta$ to verify (24) so we obtain the following conditions:

$$
\begin{aligned}
&\left(B_{11}+B_{22}\right)\left(B_{12}^{2}-B_{34}^{2}\right)=\left(B_{11}-B_{22}\right)\left(B_{24}^{2}-B_{13}^{2}\right)- \\
& 2 B_{23}\left(B_{24} B_{34}+B_{12} B_{13}\right)- \\
& 2 B_{14}\left(B_{13} B_{34}+B_{12} B_{24}\right) \\
&(\epsilon+\eta)\left(B_{34}^{2}-B_{12}^{2}\right)=(\epsilon-\eta)\left(B_{24}^{2}-B_{13}^{2}\right) .
\end{aligned}
$$

The system above represents a sufficient condition for (12) to be fulfilled. In order to become a necessary condition we have to verify if $\beta=0$ implies relation (12).

We denote:

$$
\begin{aligned}
& \mu_{2}^{\lambda}+\mu_{4}^{\lambda}=\xi \\
& \mu_{1}^{\lambda}+\mu_{3}^{\lambda}=-\xi
\end{aligned}
$$

with $\xi \neq 0$. Since $\beta$ is defined by:

$$
\beta=\mu_{1}^{\lambda} \mu_{2}^{\lambda} \mu_{3}^{\lambda}+\mu_{1}^{\lambda} \mu_{2}^{\lambda} \mu_{4}^{\lambda}+\mu_{1}^{\lambda} \mu_{3}^{\lambda} \mu_{4}^{\lambda}+\mu_{2}^{\lambda} \mu_{3}^{\lambda} \mu_{4}^{\lambda}
$$

together with (26) we obtain the following relation:

$$
\frac{1}{\mu_{1}^{\lambda}}+\frac{1}{\mu_{2}^{\lambda}}-\frac{1}{\xi+\mu_{1}^{\lambda}}+\frac{1}{\xi-\mu_{2}^{\lambda}}=0 .
$$

We have the following equivalences:

$$
\begin{aligned}
\frac{\xi+\mu_{1}^{\lambda}-\mu_{1}^{\lambda}}{\mu_{1}^{\lambda}\left(\xi+\mu_{1}^{\lambda}\right)} & =\frac{\xi+\mu_{2}^{\lambda}-\mu_{2}^{\lambda}}{\mu_{2}^{\lambda}\left(\mu_{2}^{\lambda}-\xi\right)} \\
\mu_{2}^{\lambda}\left(\mu_{2}^{\lambda}-\xi\right) & =\mu_{1}^{\lambda}\left(\xi+\mu_{1}^{\lambda}\right)
\end{aligned}
$$

This follows:

$$
\xi=-\frac{\left(\mu_{1}^{\lambda}\right)^{2}-\left(\mu_{2}^{\lambda}\right)^{2}}{\left(\mu_{1}^{\lambda}+\mu_{1}^{\lambda}\right)}=\mu_{2}^{\lambda}-\mu_{1}^{\lambda}
$$

We replace relation (30) in (26) and we obtain:

$$
\begin{aligned}
& \mu_{2}^{\lambda}+\mu_{4}^{\lambda}=\xi=\mu_{2}^{\lambda}-\mu_{1}^{\lambda} \\
& \mu_{1}^{\lambda}+\mu_{3}^{\lambda}=-\xi=\mu_{1}^{\lambda}-\mu_{2}^{\lambda}
\end{aligned}
$$

This implies:

$$
\mu_{1}^{\lambda}+\mu_{4}^{\lambda}=\mu_{3}^{\lambda}+\mu_{2}^{\lambda} .
$$

and conclusion follows.

Let us take two matrices $A$ and $B$ that verify relations (16) and (25) (see Fig. 1):

$$
\begin{aligned}
A & =\left(\begin{array}{cccc}
-5 & 0 & 0 & 0 \\
0 & 4 & 0 & 0 \\
0 & 0 & -4 & 0 \\
0 & 0 & 0 & 5
\end{array}\right), \\
B & =\left(\begin{array}{cccc}
0 & -3 & 2 & 0 \\
-3 & 0 & 0.1 & 1 \\
2 & 0.1 & 0 & 6 \\
0 & 1 & 6 & 0
\end{array}\right)
\end{aligned}
$$

We can easily observe that $[A, B] \neq 0$ and the Lie algebra generated by $i A, i B$ has dimension 15 , thus contains su(4). We have for every $\lambda \in \mathbb{R}$ the existence of $(1,3) \neq(2,4)$ with $B_{13} \neq 0, B_{24} \neq 0$, such that:

$$
\mu_{1}^{\lambda}-\mu_{3}^{\lambda}=\mu_{2}^{\lambda}-\mu_{4}^{\lambda} .
$$

Let us take another $A$ and $B$ such that relations (16)

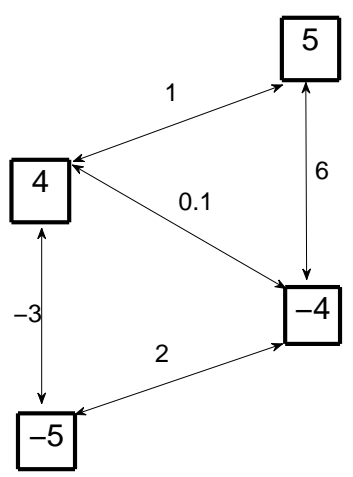

Fig. 1. Schematic view corresponding to $A$ and $B$ defined by (33). Each eigenvalue of $A$ is represented as a rectangle with the corresponding value given inside. The edges drawn between two rectangles are labeled with the corresponding value in the coupling matrix $B$.

and (25) are verified (see Fig. 2):

$$
\begin{aligned}
A & =\left(\begin{array}{cccc}
-2 & 0 & 0 & 0 \\
0 & -3 & 0 & 0 \\
0 & 0 & 3 & 0 \\
0 & 0 & 0 & 2
\end{array}\right) \\
B & =\left(\begin{array}{cccc}
2 & 1 & 1 & 0 \\
1 & 3 & 0 & 4 \\
1 & 0 & -3 & 2 \\
0 & 4 & 2 & -2
\end{array}\right)
\end{aligned}
$$

Again $[A, B] \neq 0$ and the Lie algebra generated by $i A, i B$ has dimension 15 , thus contains su(4).

- Second case: A is degenerate

We say that $A$ is degenerate if there exists $i \neq j, i, j=$ $1, \ldots, 4$ such that $\lambda_{i}^{0}=\lambda_{j}^{0}$.

Let us take

$$
\mu_{1}^{0}=\theta, \mu_{2}^{0}=-\theta, \mu_{3}^{0}=-\theta, \mu_{4}^{0}=\theta .
$$




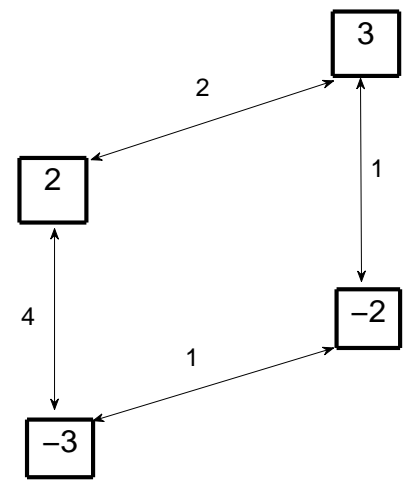

Fig. 2. Same representation as in Fig. 1 for $A$ and $B$ defined by (35).

In this case:

$$
\begin{aligned}
A & =\left(\begin{array}{cccc}
\theta & 0 & 0 & 0 \\
0 & -\theta & 0 & 0 \\
0 & 0 & -\theta & 0 \\
0 & 0 & 0 & \theta
\end{array}\right) \\
B & =\left(\begin{array}{cccc}
B_{11} & B_{12} & B_{13} & B_{14} \\
B_{12} & B_{22} & B_{23} & B_{24} \\
B_{13} & B_{23} & B_{33} & B_{34} \\
B_{14} & B_{24} & B_{34} & B_{44}
\end{array}\right)
\end{aligned}
$$

with $B_{13} \neq 0$ and $B_{42} \neq 0$.

This implies that for $\lambda=0$ equality (12) is fulfilled, since there exists $(1,3) \neq(4,2), 1 \neq 3,4 \neq 2$ with $B_{13} \neq 0, B_{24} \neq 0$ such that

$$
\mu_{1}^{0}-\mu_{3}^{0}=\mu_{4}^{0}-\mu_{2}^{0}
$$

As in the non-degenerate case we want (24) to be verified, so we obtain the following conditions:

$$
\begin{gathered}
B_{11} B_{22} B_{33}+B_{11} B_{22} B_{44}+B_{11} B_{33} B_{44}+ \\
B_{22} B_{33} B_{44}-B_{11} B_{34}^{2}-B_{22} B_{34}^{2}-B_{44} B_{23}^{2}- \\
B_{11} B_{23}^{2}-B_{44} B_{12}^{2}-B_{33} B_{12}^{2}-B_{22} B_{13}^{2}- \\
B_{44} B_{13}^{2}-B_{11} B_{24}^{2}-B_{33} B_{24}^{2}-B_{22} B_{14}^{2}- \\
B_{33} B_{14}^{2}+2 B_{23}\left(B_{24} B_{34}+B_{12} B_{13}\right)+ \\
2 B_{14}\left(B_{13} B_{34}+B_{12} B_{24}\right)=0 \\
B_{23}^{2}=B_{14}^{2}
\end{gathered}
$$

Denoting:

$$
\begin{aligned}
& \mu_{1}^{\lambda}+\mu_{2}^{\lambda}=\delta \\
& \mu_{3}^{\lambda}+\mu_{4}^{\lambda}=-\delta
\end{aligned}
$$

with $\delta \neq 0$, in the same way as in the non-degenerate case we can proof that $\beta=0$ implies relation (12).

Let us take $A$ and $B$ such that relations (16) and (39) are verified (see Fig. 3 ):

$$
\begin{gathered}
A=\left(\begin{array}{cccc}
2 & 0 & 0 & 0 \\
0 & -2 & 0 & 0 \\
0 & 0 & -2 & 0 \\
0 & 0 & 0 & 2
\end{array}\right), \\
B=\left(\begin{array}{cccc}
3 & -2 & 4 & -1 \\
-2 & -3 & 1 & 4 \\
4 & 1 & -3 & -2 \\
-1 & 4 & -2 & 3
\end{array}\right) .
\end{gathered}
$$

We can easily observe that $[A, B] \neq 0$ and the Lie algebra generated by $i A, i B$ has dimension 15 , thus contains su(4). This implies that for every $\lambda \in \mathbb{R}$ there exists $(1,3) \neq(4,2)$ with $B_{13} \neq 0, B_{24} \neq 0$, such that:

$$
\mu_{1}^{\lambda}-\mu_{3}^{\lambda}=\mu_{4}^{\lambda}-\mu_{2}^{\lambda}
$$

We have the existence of at least three matrices $A$ and $B$

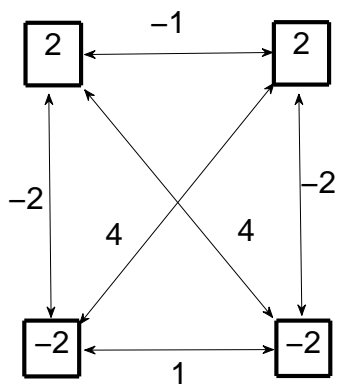

Fig. 3. Same representation as in Fig.1 for $A$ and $B$ defined by (41).

defined by (13) with conditions (16), (25) or (39), that verify the hypothesis $(i)$ and $(i i)$ such that for every $\lambda \in \mathbb{R}$ there exists $(i, j) \neq(a, b)$ with relation (12) fulfilled.

Remark 3.1: We can explain the existence of matrices $A$ and $B$, especially relation (17) for the non-degenerate case using perturbation theory [3]. We present the main idea in the following.

Denote by $H(\lambda)=A+\lambda B$, with $\lambda \ll 1$. We look for the eigenvalues $\mu(\lambda)=\left(\mu_{i}^{\lambda}\right)_{i=1, \ldots, 4}$ and the eigenvectors $\psi(\lambda)=\left(\psi_{i}\right)_{i=1, \ldots, 4}$ of the operator $H(\lambda)$ :

$$
H(\lambda)|\psi(\lambda)>=\mu(\lambda)| \psi(\lambda)>.
$$

Using the perturbation theory we suppose that $\mu(\lambda)$ can be developed in power series of $\lambda$ :

$$
\mu(\lambda)=\varepsilon_{0}+\lambda \varepsilon_{1}+\ldots \lambda^{q} \varepsilon_{q} \ldots
$$

By identification ( see [3] for more details) we have a second order development:

$$
\mu_{i}^{\lambda}=\mu_{i}^{0}+\lambda<\varphi_{i}|B| \varphi_{i}>+O\left(\lambda^{2}\right),
$$

where $\left(\varphi_{i}\right)_{i=1, \ldots, 4}$ are the eigenvectors associated to the eigenvalues $\left(\mu_{i}^{0}\right)_{i=1, \ldots, 4}$.

We replace relation (44) in (12) and we obtain:

$$
\begin{gathered}
\mu_{i}^{0}+\lambda<\varphi_{i}|B| \varphi_{i}>-\mu_{j}^{0}-\lambda<\varphi_{j}|B| \varphi_{j}>= \\
\mu_{a}^{0}+\lambda<\varphi_{a}|B| \varphi_{a}>-\mu_{b}^{0}-\lambda<\varphi_{b}|B| \varphi_{b}>.
\end{gathered}
$$

Since $\left.<\varphi_{i}|B| \varphi_{i}\right\rangle=B_{i i}$ for every $i=1, . .4$ it follows:

$$
\begin{array}{r}
\mu_{i}^{0}+\lambda B_{i i}-\mu_{j}^{0}-\lambda B_{j j}= \\
\mu_{a}^{0}+\lambda B_{a a}-\mu_{b}^{0}-\lambda B_{b b} .
\end{array}
$$

In fact by analyticity relation (46) is true for every $\lambda \in \mathbb{R}$. Let us take $\lambda=0$ and we obtain:

$$
\mu_{i}^{0}-\mu_{j}^{0}=\mu_{a}^{0}-\mu_{b}^{0} .
$$


We replace $(47)$ in $(46)$ and we have:

$$
B_{i i}-B_{j j}=B_{a a}-B_{b b} .
$$

Thus (12) implies relations (47) and (48).

\section{NUMERICAL RESUlts}

Simulations in Fig. 4, left figure describe the eigenvalues $\mu_{i}^{\lambda}, i=1, \ldots, 4$ of the matrix $A+\lambda B$ for $\lambda \in[-10,10]$, $A, B$ given by (33); the right figure shows $\mu_{1}^{\lambda}-\mu_{3}^{\lambda}-0.9$ and $\mu_{2}^{\lambda}-\mu_{4}^{\lambda}$ for $\lambda \in[-10,10]$ in order to observe numerically that $\mu_{1}^{\lambda}-\mu_{3}^{\lambda}=\mu_{2}^{\lambda}-\mu_{4}^{\lambda}$.
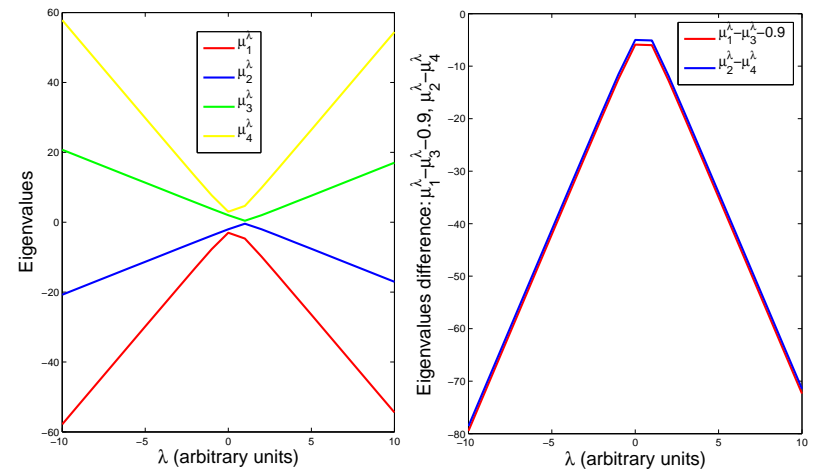

Fig. 4. Left figure: the eigenvalues of the matrix $A+\lambda B$, with $A, B$ defined by $(33) ; \lambda$ belongs to the interval $[-10,10]$; right figure: the difference of eigenvalues of the matrix $A+\lambda B, \mu_{1}^{\lambda}-\mu_{3}^{\lambda}-0.9$ and $\mu_{2}^{\lambda}-\mu_{4}^{\lambda}$, with $A, B$ defined by (33); $\lambda$ belongs to the interval $[-10,10]$.
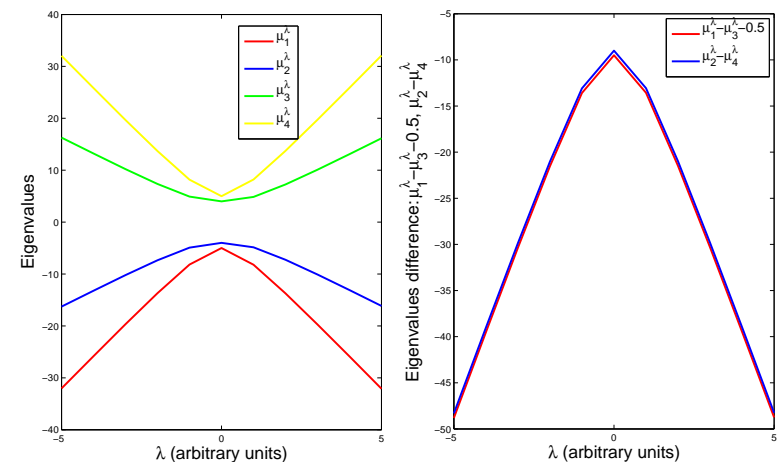

Fig. 5. Left figure: the eigenvalues of the matrix $A+\lambda B$, with $A, B$ defined by $(35) ; \lambda$ belongs to the interval $[-5,5]$; right figure: the difference of eigenvalues of the matrix $A+\lambda B, \mu_{1}^{\lambda}-\mu_{3}^{\lambda}-0.5$ and $\mu_{2}^{\lambda}-\mu_{4}^{\lambda}$, with $A, B$ defined by $(35) ; \lambda$ belongs to the interval $[-5,5]$

In Fig. 5 left image represents the eigenvalues $\mu_{i}^{\lambda}, i=$ $1, \ldots, 4$ of the matrix $A+\lambda B$ for $\lambda \in[-5,5] ; A, B$ given by (35); right image represents $\mu_{1}^{\lambda}-\mu_{3}^{\lambda}-0.5$ and $\mu_{2}^{\lambda}-\mu_{4}^{\lambda}$ for $\lambda \in[-5,5]$, in order to observe numerically that $\mu_{1}^{\lambda}-\mu_{3}^{\lambda}=$ $\mu_{4}^{\lambda}-\mu_{2}^{\lambda}$.

In Fig. 6 left image represents the eigenvalues $\mu_{i}^{\lambda}, i=$ $1, \ldots, 4$ of the matrix $A+\lambda B$ for $\lambda \in[-10,10] ; A, B$ given by (41); right image represents $\mu_{1}^{\lambda}-\mu_{3}^{\lambda}-0.9$ and $\mu_{2}^{\lambda}-\mu_{4}^{\lambda}$ for $\lambda \in[-10,10]$, in order to observe numerically that $\mu_{1}^{\lambda}-\mu_{3}^{\lambda}=$ $\mu_{4}^{\lambda}-\mu_{2}^{\lambda}$.
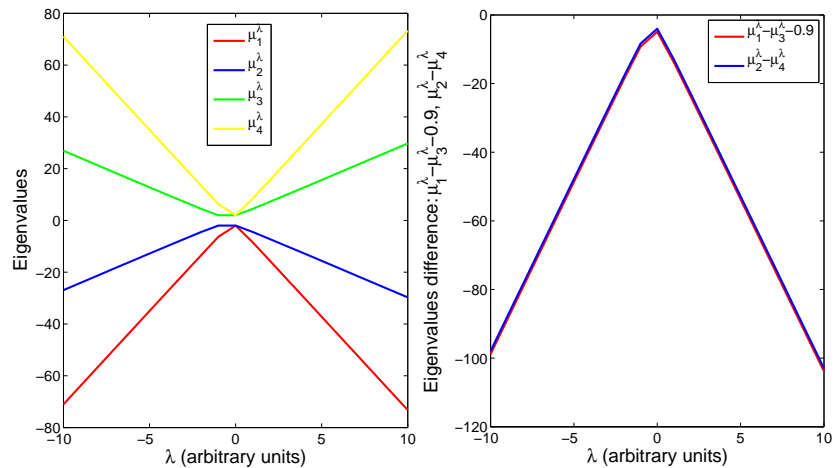

Fig. 6. Left figure: the eigenvalues of the matrix $A+\lambda B$, with $A, B$ defined by (41); $\lambda$ belongs to the interval $[-10,10]$; right figure: the difference of eigenvalues of the matrix $A+\lambda B, \mu_{1}^{\lambda}-\mu_{3}^{\lambda}-0.9$ and $\mu_{2}^{\lambda}-\mu_{4}^{\lambda}$, with $A, B$ defined by $(41) ; \lambda$ belongs to the interval $[-10,10]$.

\section{CONCLUSIONS}

In this paper, we focus on global controllability for a 4dimensional quantum system. We prove a negative result to a conjecture formulated earlier in the literature concerning the "strong regularity" cf. [1] (eventually after the introduction of a additive constant in the field) of a controllable system.

On the other hand we analyse the local controllability around a constant field. Same result apply and shows that controllable systems exists such that trajectories corresponding to constant fields are singular.

\section{REFERENCES}

[1] C. Altafini. Controllability of quantum mechanical systems by root space decomposition of su(n). J. Math. Phys., 45(5), 2002.

[2] P. Brumer and M. Shapiro. Coherent chemistry: Controlling chemical reactions with lasers. Acc. Chem. Res., 22:407, 1989.

[3] C. Cohen-Tannoudji, B. Liu, and F. Laloë. Quantum Mechanics. Wiley, 1997.

[4] M. Dahleh, H. Rabitz, and W.S. Warren. Coherent control of quantum dynamics: The dream is alive. Science, 259:1581-1589, 1993.

[5] P. Gross, D. Neuhauser, and H. Rabitz. Teaching lasers to control molecules in the presence of laboratory field uncertainty and measurement imprecision. J. Chem. Phys., 98:4557-4566, 1993.

[6] K. Kompa, M. Motzkus, H. Rabitz, and R. de Vivie-Riedle. Wither the future of controlling quantum phenomena? Science, 288:824-828, 2000.

[7] M. Q. Phan and H. Rabitz. Learning control of quantum-mechanical systems by laboratory identification of effective input-output maps. Chem. Phys., 217:389-400, 1997.

[8] H. Rabitz and S. Shi. Optimal control of selectivity of unimolecular reactions via an excited electronic state with designed lasers. Chem. Phys., 97:276-287, 1992.

[9] H. Rabitz, S. Shi, and A. Woody. Optimal control of selective vibrational excitation in harmonic linear chain molecules. J. Chem. Phys., 88:6870-6883, 1988.

[10] H. Rabitz and G. Turinici. Quantum wavefunction controllability. Chem. Phys., 267:1-9, 2001.

[11] H. Rabitz and G. Turinici. Wavefunction controllability in quantum systems. J. Phys.A., 36:2565-2576, 2003.

[12] S.A. Rice and D.J. Tannor. Control of selectivity of chemical reaction via control of wave packet evolution. J. Chem. Phys, 83:5013-5018, 1985.

[13] S. Schirmer and X. Wang. Analysis of Lyapunov method for control of quantum states. arXiv:0901.4515v2 [quant-ph], 2009.

[14] G. Turinici. Analysis of numerical simulation methods in quantum chemistry. Phd. Thesis, 2000. 